REVIEW

\title{
Maintaining high thrombectomy rates during pandemics
}

\author{
Tomas Dobrocky ${ }^{\mathrm{a}, \mathrm{b}}$, Johannes Kaesmacher ${ }^{\mathrm{a}, \mathrm{c}}$, Vitor Mendes Pereira ${ }^{\mathrm{b}}$, \\ Jan Gralla ${ }^{\mathrm{a}}$, and Urs Fischer ${ }^{\mathrm{d}}$
}

\begin{abstract}
Purpose of review
The aim of this article is to review the current literature on endovascular treatment of acute ischemic stroke in the aftermath of the coronavirus disease 2019 (COVID-19) lockdown.
\end{abstract}

\begin{abstract}
Recent findings
The outbreak of the COVID-19 has had effect of unprecedented magnitude on the social, economic and personal aspects around the globe. Healthcare providers were forced to expand capacity to provide care to the surging number of symptomatic COVID-19 patients, while maintaining a fully operating service for all non-COVID patients. The recent literature suggesting an overall decrease in acute ischemic stroke admissions as well as total number of endovascular treatments will be reviewed. Although the underlying reasons therefore remain the matter of debate, it seems that the imposed restrictions, requiring social distancing, and stopping all nonessential services, have led to a higher threshold for patients to seek medical attention, in particular in those with less severe symptoms. Thus, raising public awareness on the importance of strokes and transient ischemic attacks is even more important in the light of the current situation to avoid serious healthcare, economic consequences, and limit long term morbidity.
\end{abstract}

\section{Summary}

The priority remains maintaining a fast and efficient pre and in-hospital work-flow while mitigating nosocomial transmission and protecting the patient and the healthcare workers with appropriate personal protective equipment.

\section{Keywords}

acute ischemic stroke, coronavirus disease 2019 , endovascular treatment

\section{INTRODUCTION}

The coronavirus disease 2019 (COVID-19) caused by the severe acute respiratory syndrome coronavirus-2 (SARS-CoV-2) has been declared a pandemic on March 11th, 2020, by the WHO. As of August 17, 2020, the SARS-CoV-2 pandemic has caused 21921705 confirmed infections with 774905 fatal cases worldwide. The outbreak has had effect of unprecedented magnitude in the modern era on the social, economic, and personal aspects of our lives, and has struck different countries, provinces, and communities around the globe with different severity.

The infection has demonstrated a very broad clinical spectrum ranging from asymptomatic individuals to a more severe course requiring medical attention and even respiratory and vasopressor assistance. From a healthcare perspective, the system has been faced with a number of challenges which vary depending on the number of symptomatic cases and the local conditions. In general, the healthcare providers were forced to expand capacity to provide care to the surging number of symptomatic COVID19 patients requiring hospitalization, while maintaining a fully operating service for all non-COVID patients. This has led most hospitals to treat mainly emergency cases, while refraining from elective, nonlife-threatening procedures to manage inhospital capacities.

anniversity Institute of Diagnostic and Interventional Neuroradiology, Inselspital, Bern University Hospital, University of Bern, Bern, Switzerland, bepartment of Neuroradiology, Toronto Western Hospital, Ontario, Canada, ' ${ }^{\circ}$ Department of Interventional, Pediatric and Diagnostic Radiology and Department of Neurology, Inselspital, Bern University Hospital, University of Bern, Bern, Switzerland

Correspondence to Tomas Dobrocky, MD, Institute of Diagnostic and Interventional Neuroradiology, University of Bern, Inselspital Bern, Freiburgstrasse 8, Bern $\mathrm{CH}-3010$, Switzerland.

E-mail: tomas.dobrocky@insel.ch

Curr Opin Neurol 2020, 33:000-000

DOI:10.1097/WCO.0000000000000890 


\section{KEY POINTS}

- Prevent any delay in pre and in-hospital procedure in patients with suspicion for an AIS.

- Establish local protocols for transfer, imaging, intubation, and endovascular procedures in patients positive or pending COVID-19 status.

- Prevent or contain the spread of the novel virus by stringent use of PPE including gowns, N95 masks, face protection, and gloves.

- Maintain high effort for fast hospital referral in patients with suspected stroke by emergency medical services.

- Raise awareness among the general public, paramedics, nurses, and referring physicians on the importance to present patients with suspected AIS to a dedicated center (especially with milder symptoms) and thus prevent 'collateral damage.'

- Continuing research on the evolving subject of stroke treatment in the aftermath of the COVID outbreak.

Most countries have adopted a number of regulations, imposing restrictions to the general public, practicing social distancing, restricting or stopping all nonessential services, and banning public gatherings to prevent or contain the spread of the novel virus. In the aftermath of the lockdown, several healthcare professionals have raised the concern that the COVID-19 pandemic may negatively affect the quality of care for acute cerebro and cardiovascular conditions. This 'collateral damage' may be a result of patients not seeking medical attention for a health condition due to the fear of being infected by the virus.

\section{BACKGROUND}

COVID-19 has been reported to be an independent risk factor for acute ischemic stroke (AIS). A recent study from New York reported significantly higher rates of COVID-19 infections among AIS patients (46.3\%) as compared with patients with suspicion for stroke without imaging evidence of acute infarction $(18.3 \%)$ [1"]. A study from Wuhan, China which included 214 consecutive hospitalized patients with laboratory-confirmed diagnosis of COVID-19 reported that 11 (5\%) developed an acute ischemic stroke [2]. The SARS-CoV-2 virus may lead to a cytokine storm through angiotensin-converting enzyme 2 receptor binding, leading to a hypercoagulable state and an increased incidence of vascular thromboses [3]. The authors of a monocentric study from Wuhan, China reported a four-fold increased C reactive protein (51.1 versus $12.1 \mathrm{mg} / \mathrm{l}, P<0.05)$ and 12 -fold increased D-dimer (6.9 versus $0.5 \mathrm{mg} / \mathrm{l}$, $P<0.001)$ in COVID-positive patients with cerebrovascular disease [ $\left.4^{"}\right]$.

Although infected patients seem to carry a higher risk for cerebrovascular disease, several study groups and international medical societies have reported an overall decline of hospital admissions for acute cardio and neurovascular cases since the outbreak of the pandemic. In a French study, the number of admissions for myocardial infarction in 2020 before the lockdown differed from that in 2018-2019 by less than 10\%; however, after the start of lockdown, it decreased by $31 \%$ compared with the corresponding time window in 2018-2019 [5]. Similar trends were observed for AIS patients: a study from northwest Spain reported a decreased number of weekly mean admitted AIS patients during the pandemic (124 versus $173, P<0.001)$ [6]. Overall, the decrease in AIS presentations after the outbreak of the pandemic reported in the literature ranges from $21-40 \%$ [7-11]. In a US study, a mean decrease of $38 \%$ in new stroke diagnoses after the start of lockdown was observed (mean 1.13/day [SD 1.07] from 1.82 /day [SD 1.38], $P<0.01$ ), which was related to a $59 \%$ decline in the number of daily transfers from referral centers $(P<0.01), 25 \%$ fewer telestroke consultations $(P=0.08), \quad 55 \%$ fewer patients presenting directly to their institution by private vehicle $(P<0.01)$, and $29 \%$ fewer patients through emergency services $(P=0.09)$ [9]. The same group reported no significant change in the monthly number of strokes due to large vessel occlusion (LVO); however, the proportion of new LVOs nearly doubled in the COVID-19 period (38 versus $21 \%, P=0.01$ ).

Similar results were reported from California, where overall stroke volume decreased (mean weakly stroke alert: before 132 and 98 after the lockdown). During the same time period, more severe strokes with a higher National Institutes of Health Stroke Scale scores $(P=0.003)$, more large vessel occlusions $(P=0.03)$, and fewer stroke mimics were reported [12]. After the outbreak of the pandemic patients with suspicion for stroke were less likely to present at a comprehensive stroke center (CSC) when living outside of the CSC city [13].

\section{MECHANICAL THROMBECTOMY: MAINTAIN HIGH THROMBECTOMY RATES DURING PANDEMICS}

Almost all recent studies unanimously report a decrease of patients submitted to an endovascular therapy since the beginning of the lockdown. A national survey of centers providing mechanical 
thrombectomy in Great Britain reported a $27.7 \%$ decrease in mechanical thrombectomies performed during April 2020 compared with the first three months of the year [14]. A multicenter study including 17 dedicated neurovascular centers from six European countries, Canada, and the United States reported a $32 \%$ reduction in EVT procedures and an estimated 54-min increase in symptom onset-to-groin puncture time after confinement measures for COVID-19 pandemic were put into place [15"']. A French study including 32 centers, reported a $21 \%$ decrease in mechanical thrombectomy case, and a significant increase in delays $(+18 \mathrm{~min})$ between imaging and groin puncture, after the lockdown [16"'].

The reason for the 'COVID paradox' of decreasing AIS numbers in light of the hypercoagulable state produced by the virus, which many initially believed would lead to an overall increase in stroke cases, remains a matter of debate. Several hypotheses have been proposed. First, patients with acute neurologic symptoms are less likely to seek medical attention, in particular oligosymptomatic individuals, due to a fear to become infected. Second, referring physicians may be more restrictive to transfer patients with mild symptoms, especially elderly multimorbid patients. Third, improved air quality due to reduced pollution after the lockdown which may have a positive effect and contribute to a lower incidence. Several studies have demonstrated particulate matter less than $10 \mu \mathrm{m}$ and $\mathrm{SO}_{2}$ concentrations were independently associated with an increased risk of cardioembolic stroke $[17,18]$. In a previous report, the decrease in air pollution levels during the Beijing Olympics were associated with acute changes in biomarkers of inflammation and thrombosis, which may support this hypothesis [19].

There is a major concern that the indirect or collateral damage of COVID-19 on our systems of care may have a greater impact in stroke patients than the viral infection itself.

The aim of acute stroke care treatment remains unchanged, as timely reperfusion is the most important goal in acute stroke treatment [20]. As reported by Hajdu et al. an overall increase in symptom onsetto-groin puncture time after confinement measures for COVID-19 pandemic were put into place is alarming $\left[15^{-\prime}\right]$. As timely reperfusion is the most important modifiable factor in stroke treatment, the delay may lead to an overall long-term increase in strokerelated morbidity. We thus strongly believe that acute stroke management in AIS patients with suspected or confirmed COVID-19 infection or unknown status should not be delayed by any means. Instead, appropriate precautions need to be taken, preexisting work-flows need to be optimized, or new protocols need to be established to maintain a high standard of care and to protect healthcare workers from being infected by the virus [21"']. Personal protective equipment (PPE) is mandatory to prevent or contain the spread of the virus. In general, the infection rates in healthcare workers (HCWs) ranging from 1 to $29 \%$ have been reported in multiple studies [22-25]. In a study from England, similar rates of COVID-19 infection were reported in HCWs with and without direct patient contact [26]. The authors concluded that community-acquired disease or transmission among coworkers were more likely than nosocomial transmission from infected patients. Installment of physical barriers and the donning of N95 masks, face protection, and gloves for physicians or ancillary staff en-route are recommended to prevent any delay.

Raising public awareness on the importance of stroke and transient ischemic attacks is even more important in the light of the current situation to avoid serious healthcare, economic consequences, and limit long-term morbidity.

\section{CONCLUSION}

Despite the potentially increased risk of stroke by the coronavirus, due to its hypercoagulability and potential of endotehelial damage, an overall decrease in acute stroke presentations and reperfusion therapies has occurred (Covid paradox). The current literature suggests that recently imposed restrictions, requiring social distancing, and stopping all nonessential services, have led to a higher threshold for patients to seek medical attention, in particular in those with less severe symptoms. Thus, raising public awareness on the importance of strokes and transient ischemic attacks is even more important in the light of the current situation to avoid serious healthcare, economic consequences, and limit long-term morbidity. The priority remains maintaining a fast and efficient pre and in-hospital work-flow while mitigating nosocomial transmission and protecting the patient and the healthcare workers with appropriate PPE.

\section{Acknowledgements}

None.

\section{Financial support and sponsorship}

None.

\section{Conflicts of interest}

None related to this study. UF global PI for the SWIFT DIRECT study (Medtronic) and receives research grants from SNSF and serves as a consultant for Medtronic and Stryker. JG global PI of STAR, CEC member of the 
PROMISE study (Penumbra), PI for the SWIFT DIRECT study (Medtronic), consultancy; and receives Swiss National Science Foundation grants for magnetic resonance imaging in stroke.

\section{REFERENCES AND RECOMMENDED READING}

Papers of particular interest, published within the annual period of review, have been highlighted as:

- of special interest

m of outstanding interest

1. Belani $P$, Schefflein J, Kihira $S$, et al. COVID-19 is an independent risk factor

- for acute ischemic stroke. Am J Neuroradiol. 2020; 41: 1361-1364.

Good article on overall AIS risk in COVID-infected patients.

2. Mao L, Jin H, Wang M, et al. Neurologic manifestations of hospitalized patients with coronavirus disease 2019 in Wuhan, China. JAMA Neurol. 2020; 77: 683-690.

3. Connors JM, Levy JH. COVID-19 and its implications for thrombosis and anticoagulation. Blood 2020; 135:2033-2040.

4. Li Y, Li M, Wang M, et al. Acute cerebrovascular disease following COVID-19:

- a single center, retrospective, observational study. Stroke Vasc Neurol. 2020; 5: 279-284.

Good study reporting the hypercoagulable state associated with COVID infections.

5. Lantelme $\mathrm{P}$, Targe SC, Metral P, et al. Worrying decrease in hospital admissions for myocardial infarction during the COVID-19 pandemic. Arch Cardiovasc Dis. 2020; 113: 443-447.

6. Meza HT, Lambea Gil A, Saldaña AS, et al. Impact of COVID-19 outbreak on ischemic stroke admissions and in-hospital mortality in north-west Spain. Int J Stroke 2020; 1-8.

7. Uchino K, Kolikonda MK, Brown D, et al. Decline in stroke presentations during COVID-19 surge. Stroke 2020; 51:2544-2547.

8. Rinkel LA, Prick JC, Slot RE, et al. Impact of the COVID-19 outbreak on acute stroke care. J Neurol. 2020 Jul 20;1-6. https://doi.org/10.1007/s00415020-10069-1.

9. Siegler JE, Heslin ME, Thau L, et al. Falling stroke rates during COVID-19 pandemic at a comprehensive stroke center. J Stroke Cerebrovasc Dis 2020; 29:104953.

10. Desai SM, Guyette FX, Martin-Gill C, et al. Collateral damage: impact of a pandemic on stroke emergency services. J Stroke Cerebrovasc Dis 2020; 29:104988.

11. Hoyer C, Ebert A, Huttner HB, et al. Acute stroke in times of the COVID-19 pandemic: a multicenter study. Stroke 2020; 51:2224-2227.
12. Nguyen-Huynh MN, Tang $X N$, Vinson $D R$, et al. Acute stroke presentation, care, and outcomes in community hospitals in northern California during the COVID-19 pandemic. Stroke. 2020;51: 2918-2924.

13. Jasne AS, Chojecka $P$, Maran I, et al. Stroke code presentations, interventions, and outcomes before and during the COVID-19 pandemic. Stroke. 2020; 51 : 2664-2673.

14. McConachie $D, M c C o n a c h i e ~ N$, White $P$, et al. Mechanical thrombectomy for acute ischaemic stroke during the COVID-19 pandemic: changes to UK practice and lessons learned. Clin Radiol. 2020; 75: 795.

15. Hajdu SD, Pittet V, Puccinelli F, et al. Acute stroke management during the

-. COVID-19 pandemic. Stroke 2020; 51:2593-2596.

Excellent multicentric study on the decreasing AIS numbers and increasing symptom onset-to-groin puncture time.

16. Kerleroux $B$, Fabacher $T$, Bricout $N$, et al. Mechanical thrombectomy for acute

I. ischemic stroke amid the COVID-19 outbreak: decreased activity, and increased care delays. Stroke 2020; 2012-2017.

Excellent multicentric study from France (32 centers) with numbers showing alarming delays in-patient care.

17. Chung JW, Bang OY, Ahn K, et al. Air pollution is associated with ischemic stroke via cardiogenic embolism. Stroke 2017; 48:17-23.

18. Zhang $R$, Liu G, Jiang $Y$, et al. Acute effects of particulate air pollution on ischemic stroke and hemorrhagic stroke mortality. Front Neurol 2018; 9:1-7.

19. Rich DQ, Kipen HM, Huang W, et al. Association between changes in air pollution levels during the Beijing Olympics and biomarkers of inflammation and thrombosis in healthy young adults. JAMA 2012; 307:2068-2078.

20. Saver JL, Goyal $M$, van der Lugt $A$, et al. Time to treatment with endovascular thrombectomy and outcomes from ischemic stroke: a meta-analysis. JAMA 2016; 316:1279-1288.

21. Qureshi Al, Abd-Allah F, Alsenani F, et al. Management of acute ischemic

- stroke in patients with COVID-19 infection: report of an international panel. Int J Stroke 2020; 15:540-554.

Excellent article on overall management of AIS patients during the pandemic.

22. Wu Z, McGoogan JM. Characteristics of and important lessons from the coronavirus disease 2019 (COVID-19) outbreak in China: summary of a report of 72314 cases from the Chinese Center for Disease Control and Prevention. JAMA. https://doi.org/10.1001/jama.2020.2648.

23. Wong SC, Kwong RT, Wu TC, et al. Risk of nosocomial transmission of coronavirus disease 2019: an experience in a general ward setting in Hong Kong. J Hosp Infect 2020; 105:119-127.

24. Kluytmans-van den Bergh MF, Buiting AG, Pas SD, et al. Prevalence and clinical presentation of healthcare workers with symptoms of coronavirus disease 2019 in 2 Dutch hospitals during an early phase of the pandemic. JAMA Netw Open 2020; 3:e209673.

25. CDC COVID-19 Response Team. Characteristics of healthcare personnel with COVID-19: United States, February 12-April 9, 2020. MMWR Morb Mortal Wkly Rep 2020; 69:477-481.

26. Hunter E, Price DA, Murphy E, et al. First experience of COVID-19 screening of health-care workers in England. Lancet (London, Engl) 2020; 395:e77-e78. 